\title{
Examples of application of tensorial resistivity probability tomography to architectonic and archaeological targets
}

\author{
Boris Di Fiore $\left({ }^{1}\right)$, Paolo Mauriello $\left({ }^{2}\right)$, Dario Monna $\left({ }^{2}\right)$ and Domenico Patella $\left({ }^{3}\right)$ \\ $\left.{ }^{1}{ }^{1}\right)$ Dipartimento di Geofisica e Vulcanologia, Università «Federico II», Napoli, Italy \\ (2) Istituto per le Tecnologie Applicate ai Beni Culturali, Consiglio Nazionale delle Ricerche, Roma, Italy \\ $\left.{ }^{3}\right)$ Dipartimento di Scienze Fisiche, Università «Federico II», Napoli, Italy
}

\begin{abstract}
We present the results of the application of the tensor resistivity method to the assessment of the state of conservation of some architectonic features of the S. Giovanni a Carbonara monumental monastery (Naples, Italy) and to the recognition of buried remains in the archaeological site of the roman Port of Claudius at Fiumicino (Rome, Italy). The 3D tomographic approach, based on the concept of resistivity anomaly source occurrence probability, has been used for the analysis of the invariant parameter related to the trace of the determinant of the apparent resistivity tensor.
\end{abstract}

Key words applied geoelectrics-apparent resistivity tensor - probability tomography - cultural heritage

\section{Introduction}

High-resolution acquisition techniques favour the application of $3 \mathrm{D}$ tomographic imaging in non-invasive microgeophysical testing for monument preservation and in nearsurface geophysical prospecting for archaeology. In particular, probability tomography is gaining increasing importance for its objectivity and reliability. It has the notable advantage of not requiring any a priori assumption about the structures to be imaged. The theoretical principles

Mailing address: Prof. Domenico Patella, Dipartimento di Scienze Fisiche, Università «Federico II», Complesso Universitario Monte S. Angelo, Via Cintia, 80126 Napoli, Italy; e-mail: patella@na.infn.it of 3D probability tomography were originally established for the self-potential method (Patella, 1997a,b) and then extended to the geoelectrical method for both the scalar (Mauriello et al., 1998; Mauriello and Patella, 1999) and tensor (Mauriello and Patella, 2001) apparent resistivity acquisition modes.

The aim of this paper is to illustrate the results of the application of the Tensorial Resistivity Probability Tomography (TRPT) method to the assessment of the conservation state of a few architectural features in the San Giovanni a Carbonara ancient monastery (Naples, Italy) and the recognition of a marble cargo sunk in the roman Port of Claudius at Fiumicino (Rome, Italy).

\section{Methodological synopsis}

In performing a resistivity survey on a flat surface $S$, identified by a reference $(x, y)$ plane, the adoption of two current bipoles (fig. 1) allows 


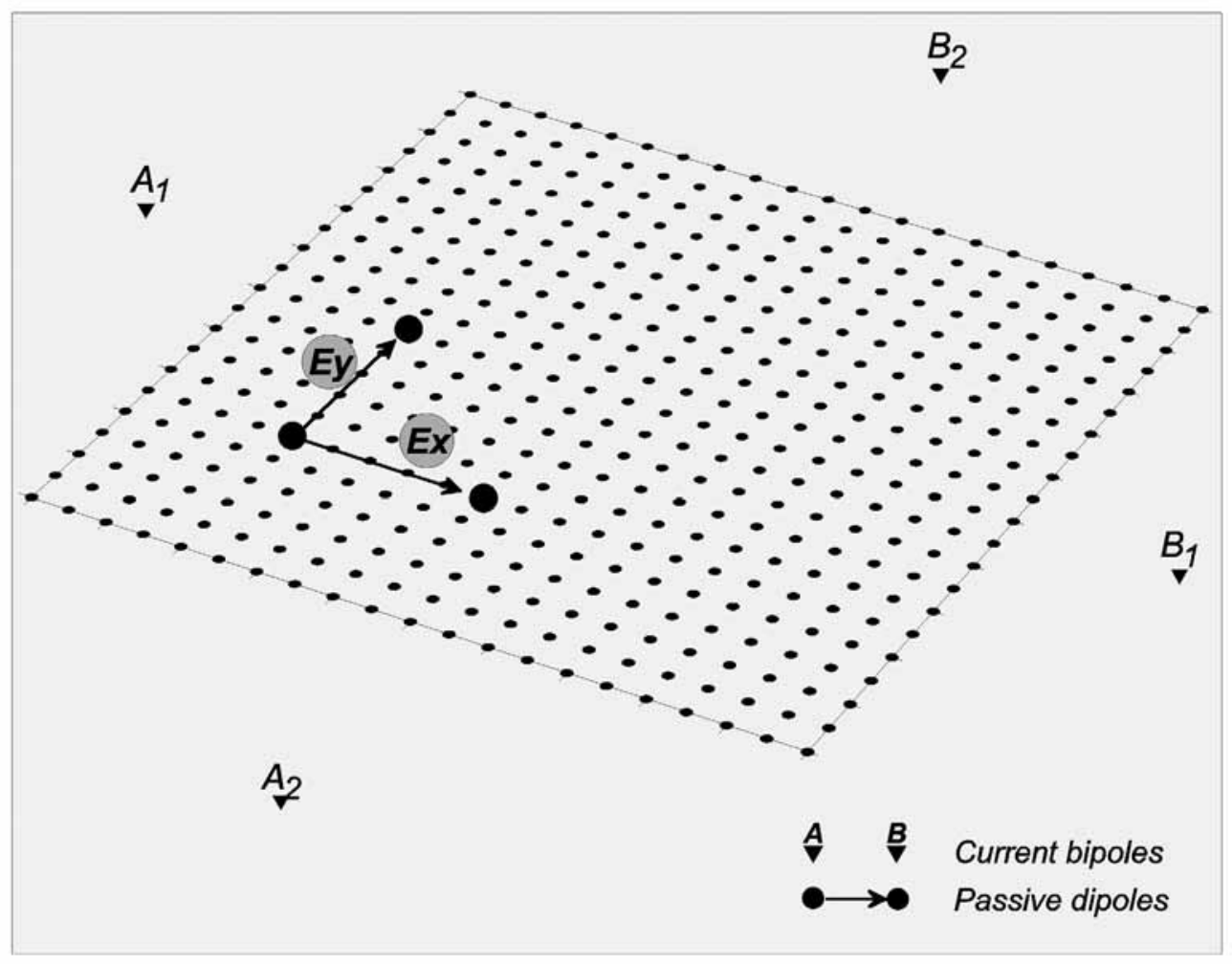

Fig. 1. A schematic depiction of the high-resolution $2 \mathrm{D}$ acquisition procedure for the determination of the matrix elements of the apparent resistivity tensor.

an apparent resistivity tensor $\rho^{(a)}$ to be determined in terms of the $x$ and $y$ components of the electric field $\boldsymbol{E}_{i}$ and the primary current density $\boldsymbol{J}_{i}$, with $i=1,2$ indicating the active bipole (Bibby, 1977). In order to obtain structural information independent of the electric field direction and the location of the exciting current bipole, tensor invariants $P$ can be deduced from $\rho^{(a)}$. We consider the invariant $P$ related to the trace of $\rho^{(a)}$ (Bibby, 1986).

The TRPT method is based on an integral elaboration of the whole dataset of the trace of $\rho^{(a)}$, and aims at imaging the most probable location of the sources of the observed apparent resistivity anomalies through the analysis of a Resistivity Anomaly Occurrence Probability (RAOP) function (Mauriello and Patella, 2001). The RAOP function is formally derived from the partition of the expression of the total geoelectrical signal power on the surface into a set of elementary contributions. The investigated volume is, in fact, divided into a set of $Q$ cells with sufficiently small volume, and each cell is assumed to contribute to the observed total signal. The 3D TRPT method consists in a crosscorrelation between a scaled version of the collected data set $\Delta P=P-P_{0}$ and the scanner function $\mathfrak{J}_{q}=\partial P_{0} / \partial \rho_{q} \cdot P_{0}$ is the tensor invariant for a reference homogeneous half-space and $\partial P_{0} / \partial \rho_{q}$ is the Frechet derivative of $P_{0}$ for a perturbation of the half-space resistivity in the $q$ th cell $(q=1,2, \ldots \ldots, Q)$. The RAOP function $\eta_{q}$ is defined as

$$
\eta_{q}=C_{q} \sum_{s} \Delta P \cdot \Im_{q}
$$




\section{$\mathrm{B}_{2}$}

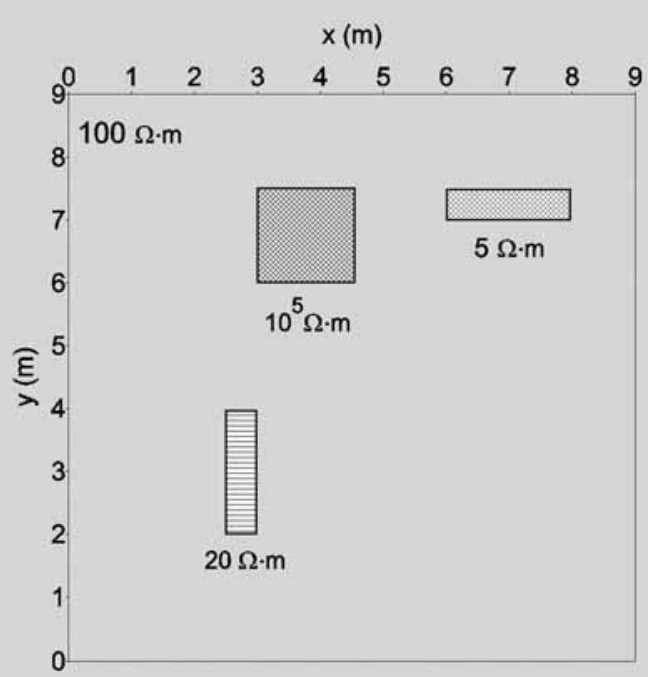

$\mathrm{A}_{1}$

(a)
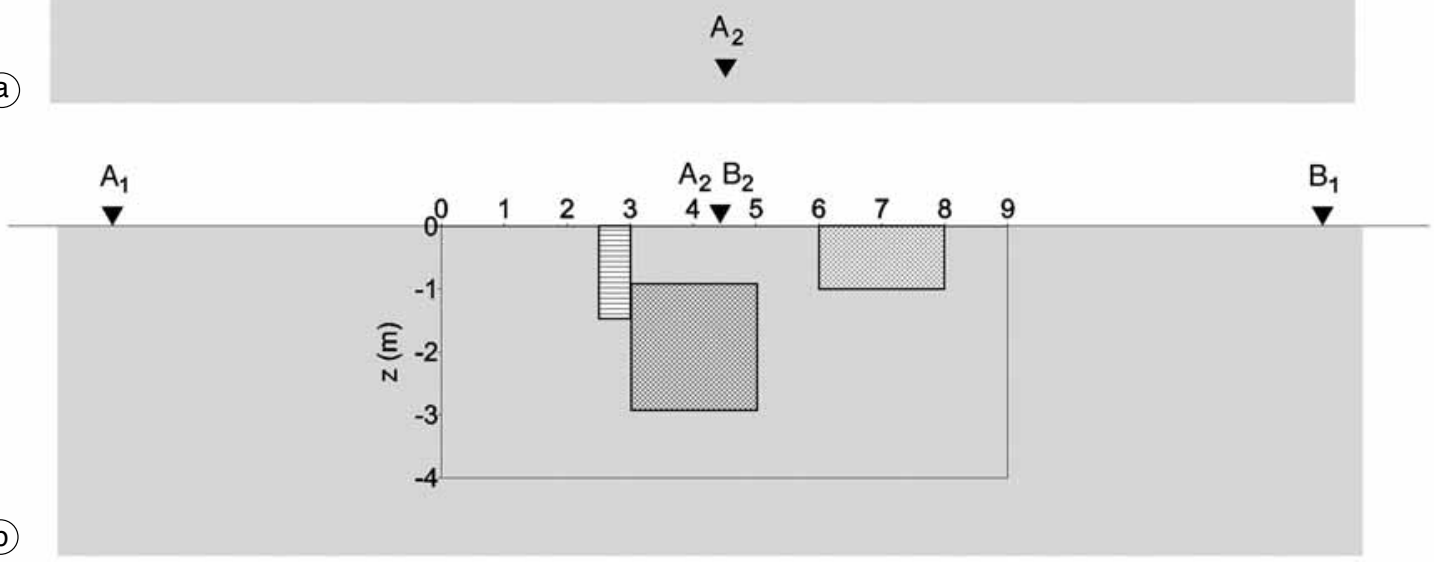

Fig. 2a,b. A synthetic three-prism resistivity model: a) plan view; b) section view. 


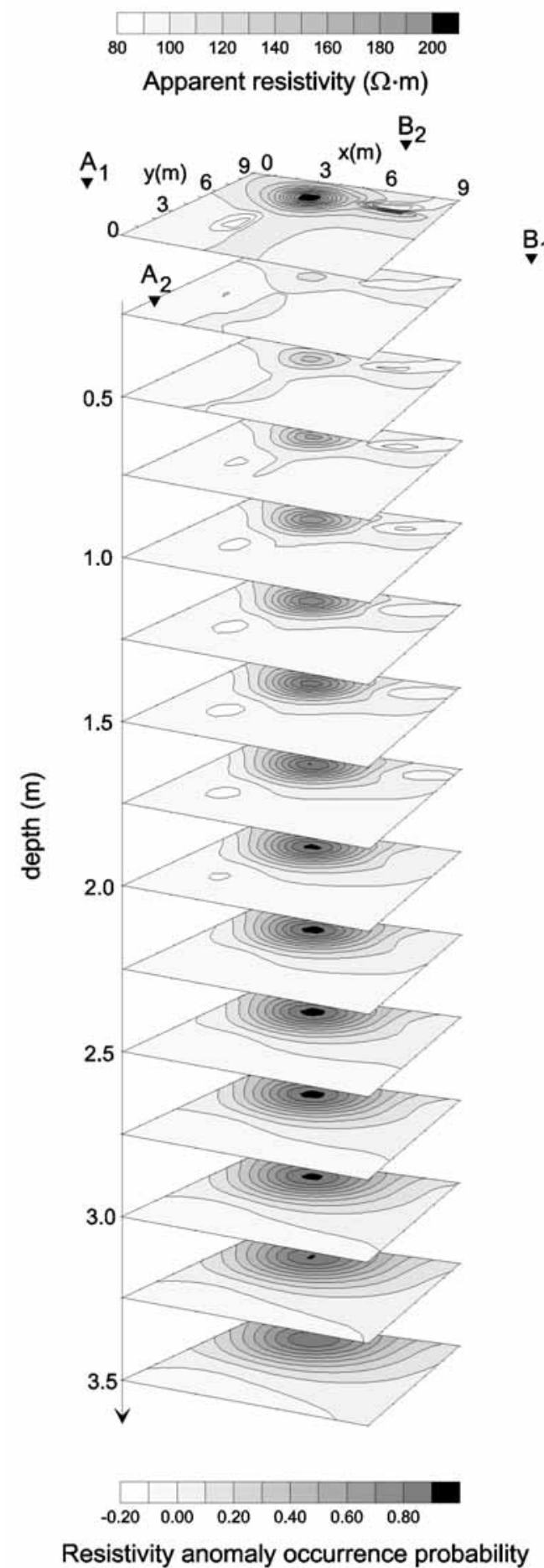

where the normalisation factor $C_{q}$ is given by

$$
C_{q}=1 / \sqrt{\Sigma_{s} \Delta P^{2} \cdot \Sigma_{s} \mathfrak{\Im}_{q}^{2}}
$$

Each $\eta_{q}$ value is interpreted as the probability with which a resistivity increment $\left(\eta_{q}>0\right)$ or decrement $\left(\eta_{q}<0\right)$ in the $q$ th cell, with respect to the selected reference resistivity, can be considered responsible for the whole observed apparent resistivity data set. An elementary cell with unitary strength is used to scan the whole target-space in order to search where resistivity variations with respect to the chosen reference resistivity are located in probabilistic sense.

Before showing the results of the application of the TRPT method to real cases, we illustrate the whole procedure by examining a synthetic model. The model consists of three prismatic blocks with resistivities of 5,20 and $10^{5} \Omega \cdot \mathrm{m}$, respectively, buried in a uniform half-space with a resistivity of $100 \Omega \cdot \mathrm{m}$. We assume that the $10^{5}$ $\Omega \cdot \mathrm{m}$ block represents the target and that the two other blocks represent shallow disturbing bodies. Figure 2a,b shows a plan and a section view of the three-prism model in the upper and lower pictures, respectively. $A_{1} B_{1}$ and $A_{2} B_{2}$ is a pair of orthogonal current bipoles used to simulate an apparent resistivity tensor survey. A standard finite element program has been edited to draw the synthetic response due to this model. Each current bipole is $19 \mathrm{~m}$ long, and a passive $0.5 \mathrm{~m}$ long dipole is assumed to be moved at steps of $0.5 \mathrm{~m}$ along crossed profiles spaced $0.5 \mathrm{~m}$ apart, within a survey area of $9 \times 9 \mathrm{~m}^{2}$. A total of 361 apparent resistivity data were processed to obtain the TRPT simulation.

The $100 \Omega \cdot \mathrm{m}$ background resistivity was taken as the uniform resistivity of the reference homogeneous half-space. The results of the TRPT imaging are depicted in fig. 3. The top slice in fig. 3 shows the anomaly map of the

Fig. 3. Tomographic images of the resistivity anomaly occurrence probability for the three-prism model of fig. 2a,b. 
invariant $P$, and the following slices show the relative TRPT imaging results. The TRPT slices display two weak shallow negative RAOP nuclei in correspondence with the two disturbing bodies, and a deeper, more intense positive RAOP nucleus in the range 1-3 m, closely in correspondence with the position of the target body. The probability image of the resistive prism appears much better delineated than the images of the two conductive bodies, due to its greater resistivity contrast with the background material.

\section{Examples of application}

We show now the results of the application of the 3D TRPT method to the study of some architectural features in the monumental monastery of San Giovanni a Carbonara (SGC), located in the historical centre of Naples, and to the recognition of a marble cargo sunk in the ancient Roman Port of Claudius, located in an area outside the terminals of the international airport of Fiumicino (Rome).

\subsection{San Giovanni a Carbonara at Naples}

The construction of the SGC complex started in 1344 , based on a plan that has been expanded and redrawn in the course of the centuries, before reaching the present structure (fig. 4). The monastery was definitively locked in 1799. Then, the building was used to house military hospitals, boards and barracks. In recent times, it has also housed offices of the public attorney, which have been now completely removed. All these events have caused a complex and articulated architectural bedding, as well as a high deterioration of the primal, very valuable structures.

In the frame of a total restoration project led by the Neapolitan Authority for Architectonic and Environmental Property, geoelectrical (GE) analyses were included within the preliminary studies aimed at assessing the state of conservation of the monument. The SGC monastery has, in fact, been retained as an ideal laboratory to test accuracy and reliability of scaled down GE methods applied to architectonic heritage, using a new type of absolutely non-invasive capacitive electrodes.

In particular, the aims of the GE tests in the SGC complex were to help to diagnose the conservation state of the stone slab facing of one of the pillars supporting the arcades of the balcony at the first floor of the 16th century Pietra Serena cloister, and to locate a blind valuable architectural feature along a plastered portion of wall at the first floor of the same cloister.

\subsubsection{Pietra Serena cloister: the experiment on the pillar}

The GE measurements were carried out on two adjacent faces of a pillar of the Pietra Serena cloister, inside an area of $1 \times 1 \mathrm{~m}^{2}$ on each face. A $128 \mathrm{~Hz}$ alternating current was injected using two perpendicular energizing bipoles, and the potential differences were measured between any two adjacent points spaced $0.5 \mathrm{~cm}$ inside a $2 \mathrm{D}$ regular grid (fig. 1). The potential differences were measured in both the parallel and perpendicular directions with respect to the bipole axis, for both bipole orientations, in order to estimate the matrix elements of the apparent resistivity tensor. In each survey face, the average value of all the determinations of the tensor invariant has been assumed as the reference value for the computation of the RAOP function in the TRPT imaging.

The pillars have an original facing consisting of slabs of Pietra Serena, a typical Tuscan sandstone, successively covered by grout beds, and an internal structure made of mortars and blocks of various materials. Figures 5a and 6a show the contour maps of the selected tensor invariant, obtained on the two adjacent faces. The TRPT images were drawn on a sequence of vertical slices up to a penetration of $20 \mathrm{~cm}$ from the freeface (figs. $5 b$ and $6 b$ ).

The first remarkable feature appearing in the tomographies of figs. $5 \mathrm{~b}$ and $6 \mathrm{~b}$ is the presence of well defined elongated positive RAOP spots, which show the highest values on the slice at $12.5 \mathrm{~cm}$ penetration. These alignments are interpreted as portions of vertical and horizontal contact zones between adjacent slabs of Pietra Serena. The occurrence of positive RAOP values 


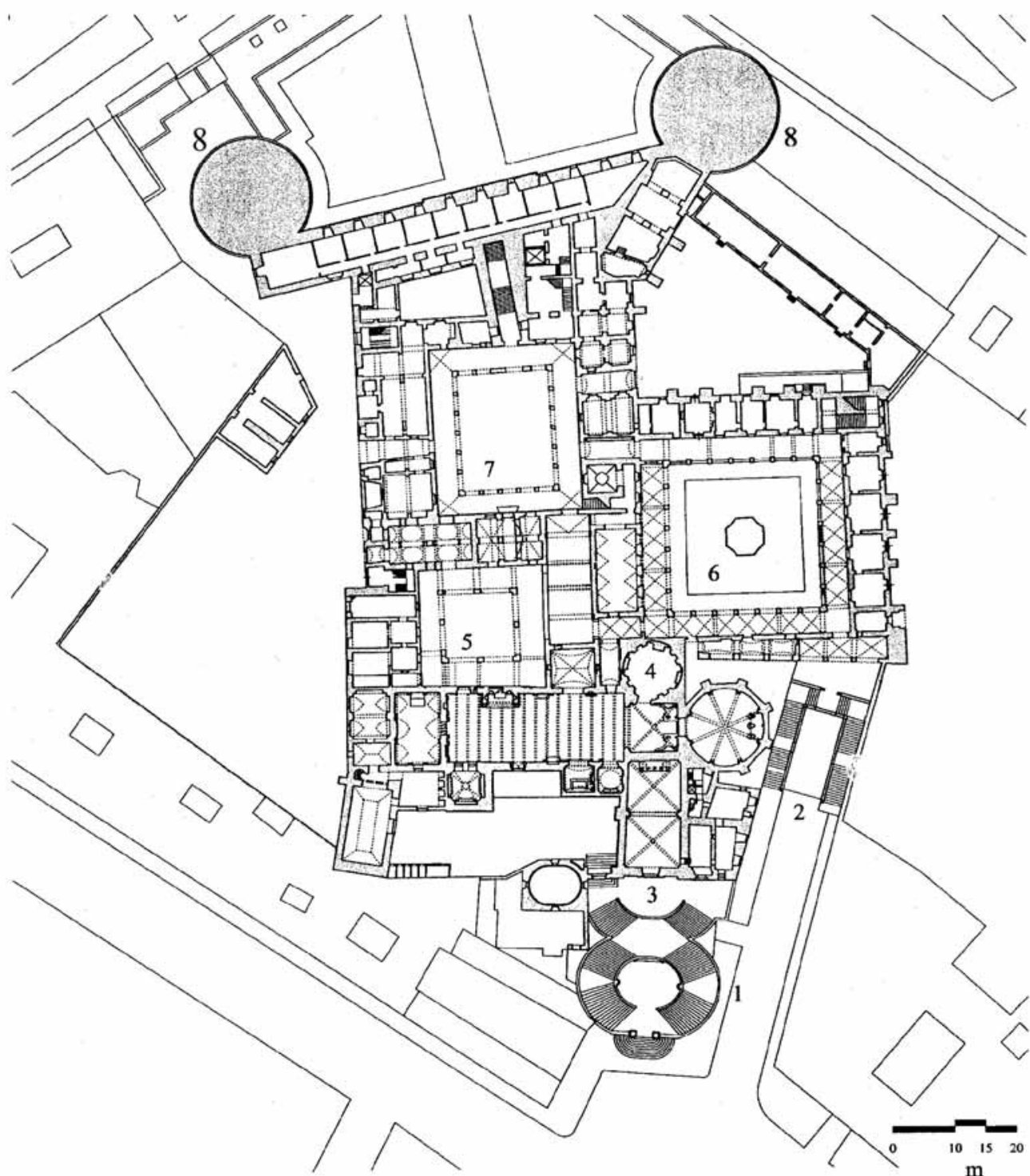

1. Sanfelice Steps

2. Seripando grand staircase

3. St. Mary Church

4. Caracciolo di Vico Chapel
5. King Ladislao Cloister

6. Pietra Serena Cloister

7. New Cloister

8. Towers of the ancient Aragonese boundary walls

Fig. 4. Structural plan of the San Giovanni a Carbonara monumental monastery, located in the historical centre of Naples, Italy. 


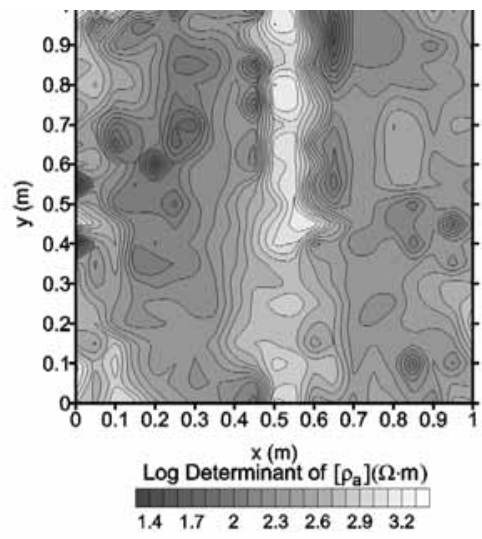

(a)

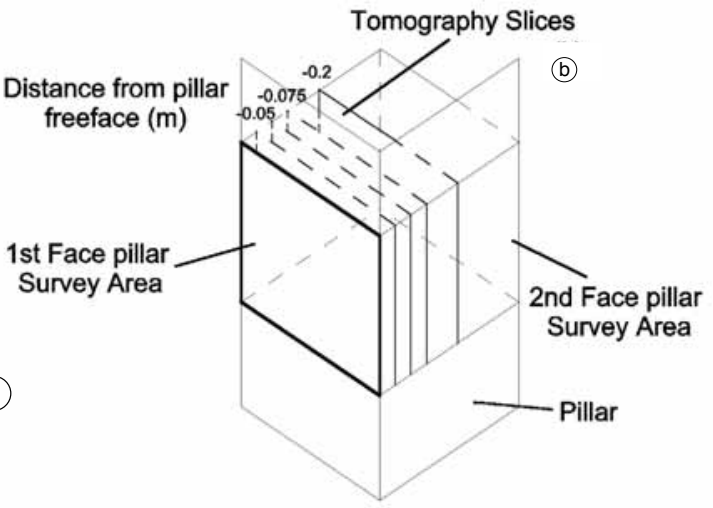

(b)

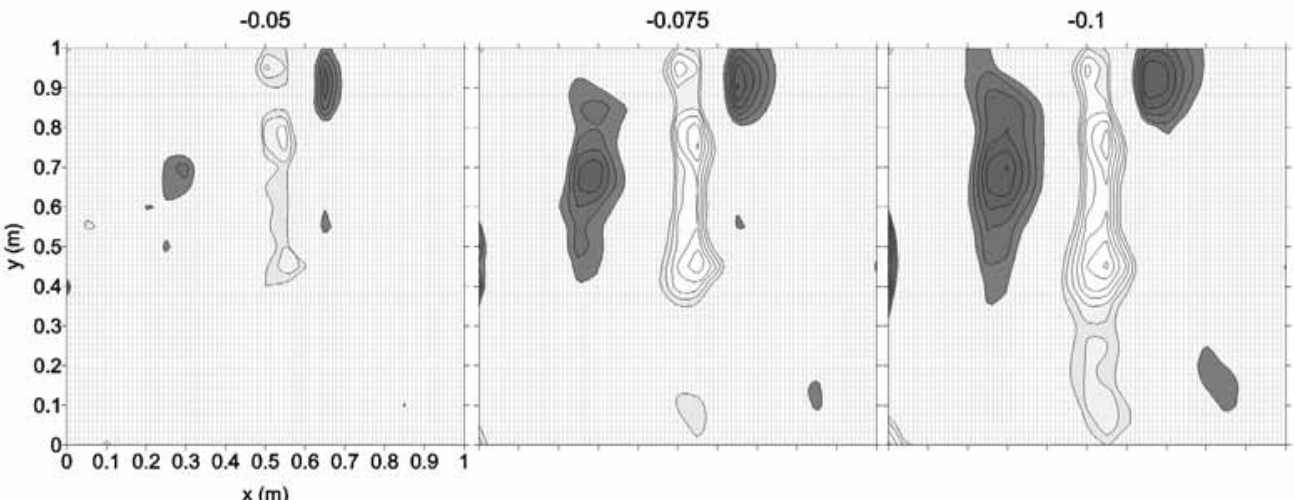

$x(m)$

$-0.125$

$-0.15$

$-0.2$

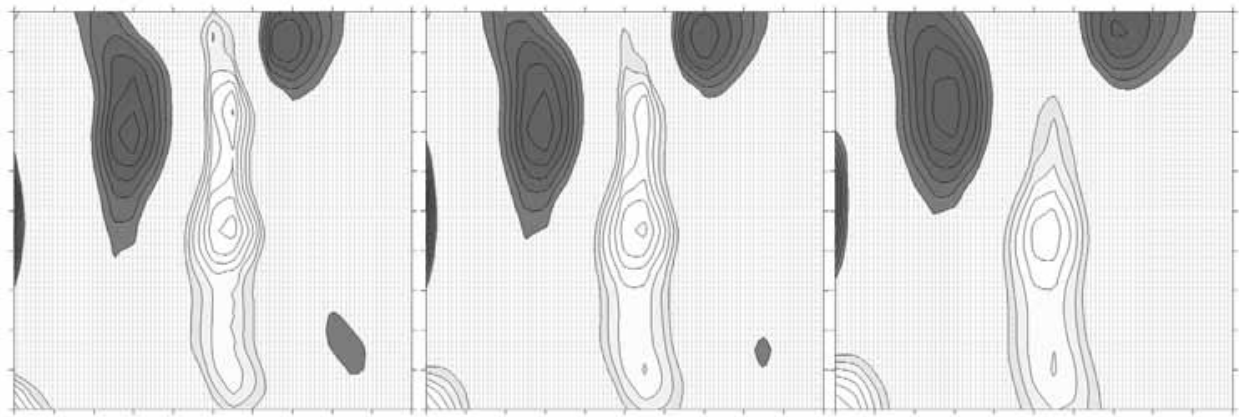

Resistivity anomaly occurrence probability

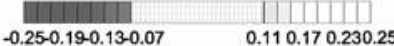

Fig. 5a,b. The tensor resistivity probability tomography applied to the first face of the selected pillar in the Pietra Serena Cloister at San Giovanni a Carbonara (Naples, Italy). a) The surface map of the invariant parameter related to the trace of the determinant of the apparent resistivity tensor. b) A sequence of tomographic slices at increasing penetration. 


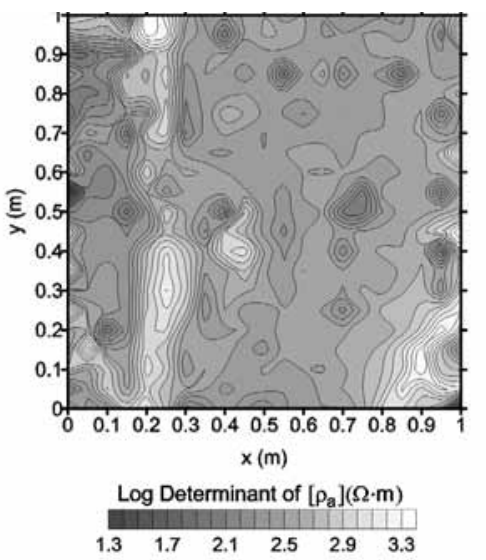

$-0.05$
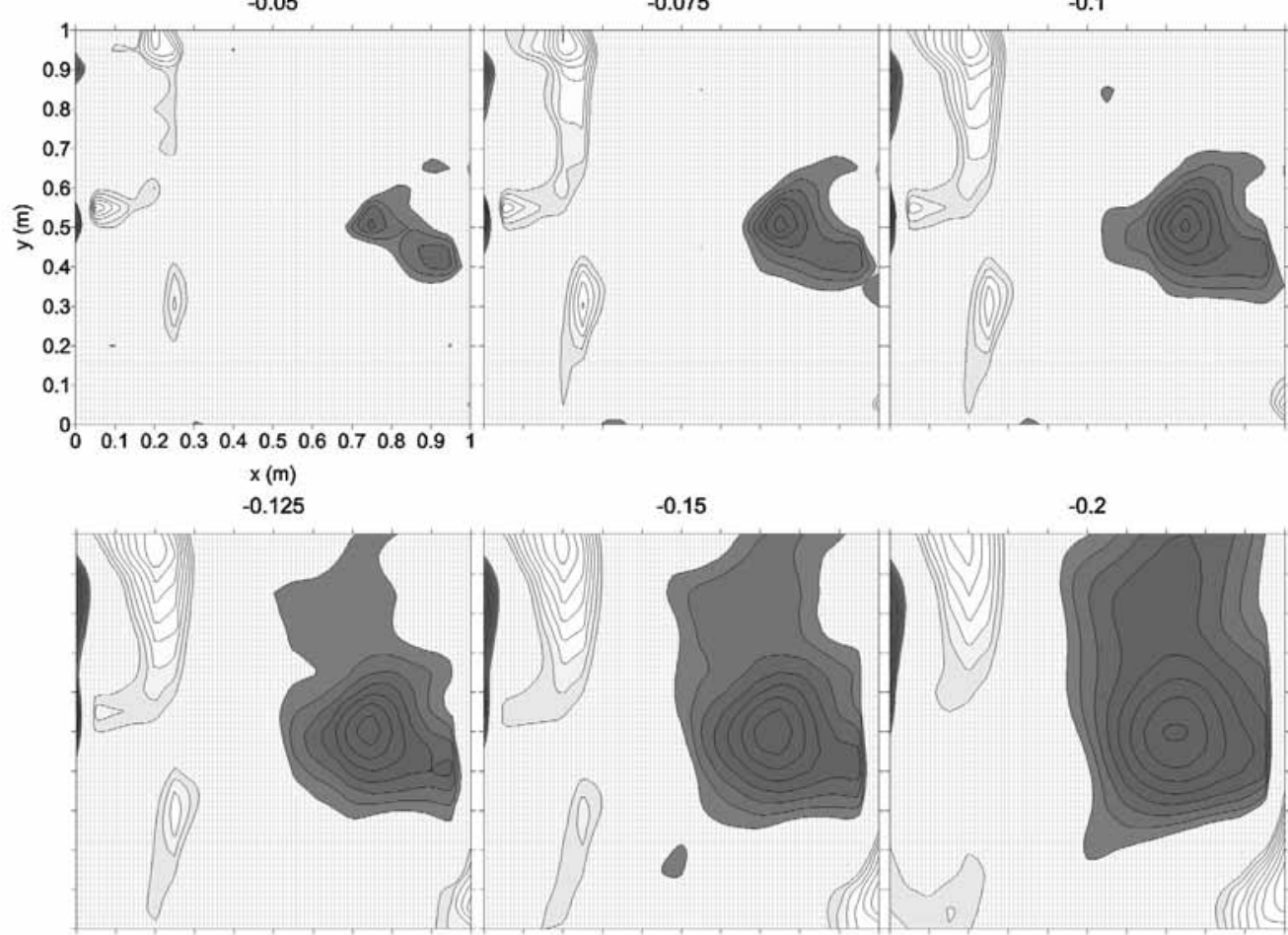

Resistivity anomaly occurrence probability

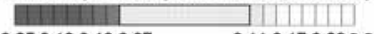

(b)

$-0.075$

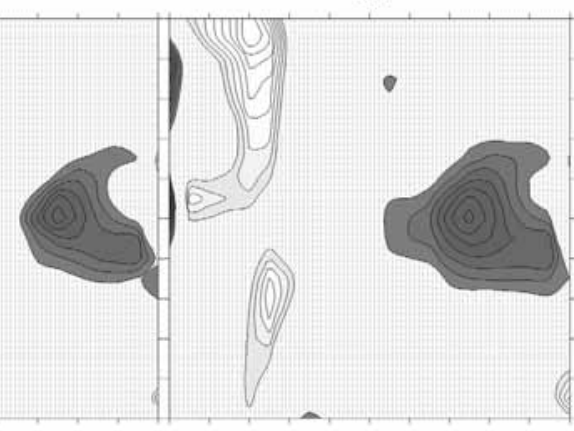

$-0.2$

2nd Face pillar Survey Area

1st Face pillar omography Slices

(b)

Distance from pillar freeface $(\mathrm{m})$

(a)

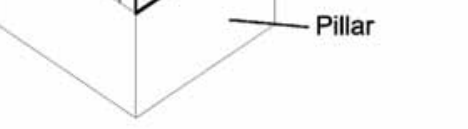

0.1

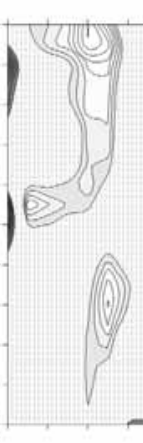


is explained by admitting a breaking up of the pasting mortars. The local increase in resistivity is, in fact, fully compatible with the presence of void fissures between adjacent slabs. The second remarkable feature is the presence of either elongated or rounded negative RAOP spots, which show the lowest values starting from 15 $\mathrm{cm}$ up to $20 \mathrm{~cm}$, at least. These negative spots can be explained as due to condensation of humidity just behind the Pietra Serena slabs, or the presence of alteration zones of the same slabs, or a combination of both factors.

\subsubsection{Pietra Serena cloister: the experiment on the wall}

The GE survey was carried out on an almost totally plastered portion of wall at the first floor of the Pietra Serena cloister inside an area of $2 \times 2 \mathrm{~m}^{2}$. This survey had been required in order to locate one of the external edges of the Caracciolo di Vico chapel, inglobed in the wall of the Pietra Serena cloister during the Renaissance. It consists of a block of Piperno, a typical welded, perlitic pyroclastic stone, inserted into blocks of Neapolitan Yellow Tuff, constituting the Pietra Serena cloister wall all around. We used the same acquisition procedure as in the previous example (fig. 1). Figure 7a shows the contour map of the selected tensor invariant obtained over the wall portion. The TRPT images have again been drawn on a sequence of vertical slices up to a penetration of $20 \mathrm{~cm}$ from the freeface (fig. $7 \mathrm{~b}$ ).

The first remarkable feature appearing in the tomographies of fig. $7 \mathrm{~b}$ is the presence of a well defined squared sequence of negative RAOP spots in the central lower sector of the slices, which reach the highest values on the slice at $20 \mathrm{~cm}$ of penetration. This squared sequence is interpreted as the signature of the boundary of a Piperno block of about $60 \mathrm{~cm}$ of height and $90 \mathrm{~cm}$ of width. The contact zones of the Piperno block with the surrounding Yellow Tuff wall are, in fact, preferential paths for humidity to rise by capillarity from the ground and cause a local decrease of resistivity. Another negative RAOP spot appears in the central top part of the slices in a zone where the wall had been exposed and presented clear signs of humidity along an injection of mortar seaming two adjacent Yellow Tuff blocks. The second remarkable feature is the presence of a continuous positive RAOP anomaly at the right-hand margin of the slices, from top to bottom. The presence of positive RAOP values in this area can be explained by admitting a local compactness of the Yellow Tuff wall.

\subsection{Port of Claudius at Fiumicino (Rome)}

During the construction of the international airport of Fiumicino (Rome), marble blocks and planking were dug out suggesting the presence of a cargo transported by one of several boats, which possibly sunk near the right mole of the roman Port of Claudius. A geophysical intervention was required to help identify the sunken ships, through the localisation of the cargo, now buried in the sediments piled up in the area due to a sea water regression of about $3 \mathrm{~km}$ (see fig. 8).

Among the various geophysical methods, the GE method was deemed the best suited to differentiate the container (sediments) from the content (transported lapidary material) buried at approximately $2 \mathrm{~m}$ of depth. Again, the same technique depicted in fig. 1 was adopted with $0.5 \mathrm{~m}$ long receiving dipoles. Figure 9a shows the survey map of the selected apparent resistivity tensor invariant. In this map, which was the largest area that could be prospected, the black circles indicate the points where some archaeological material was dug out by chance inside a trench of $3 \times 3 \mathrm{~m}^{2}$ cross-section, which was excavated along the longer median axis of the surveyed rectangle and then filled with reworked sediments. In particular, the circle at the left-hand side indicates the point where some marble remnants were found, while the circle on the right-hand side indicates the point where planking was dug out.

The most notable features appearing in the tomographies of fig. $9 \mathrm{~b}$ are the presence of a sequence of negative nuclei along the main axis of the surveyed rectangle, and a small positive nucleus located about the lower left-hand corner of the area. Both anomalies reach the highest absolute probability values around $3 \mathrm{~m}$ of depth. 

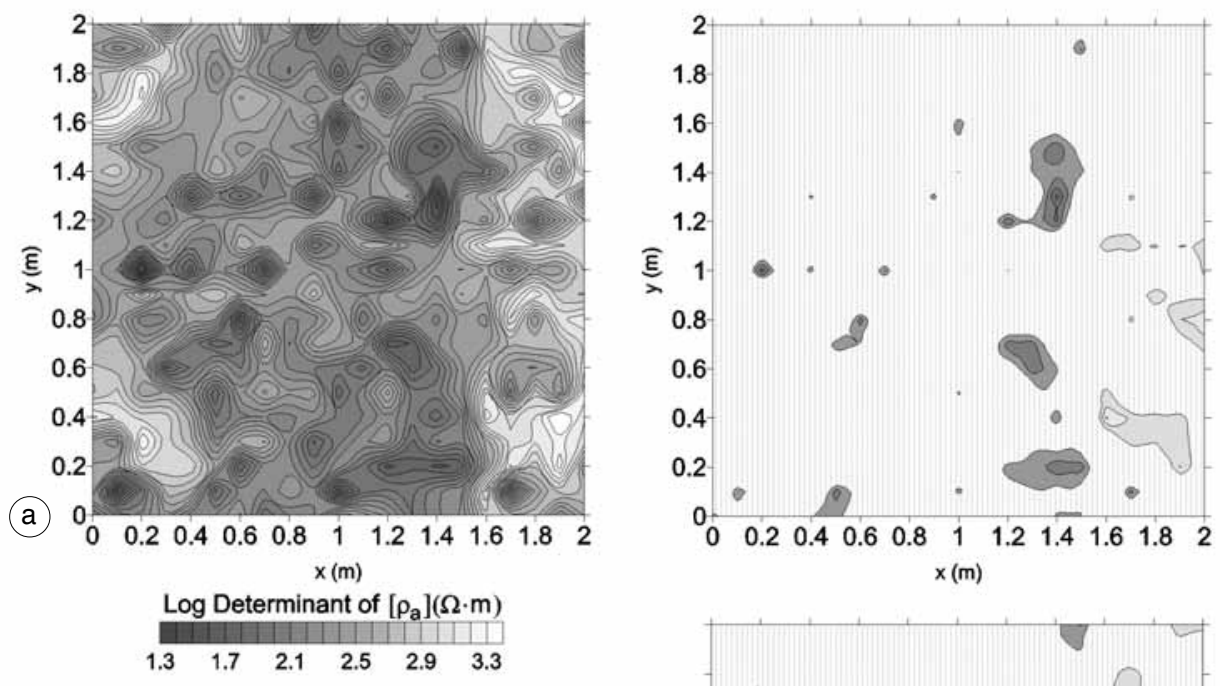

(b)

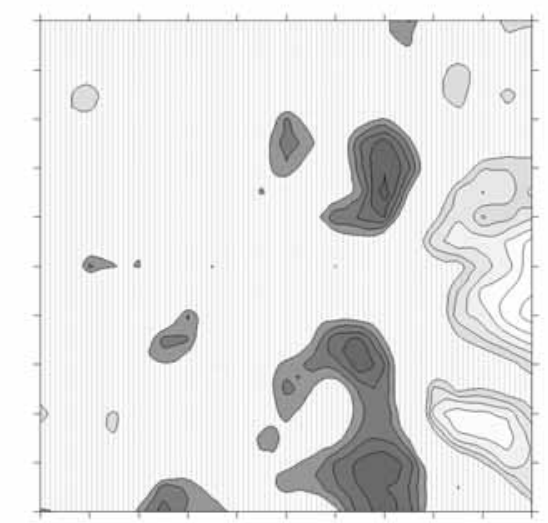

(b)
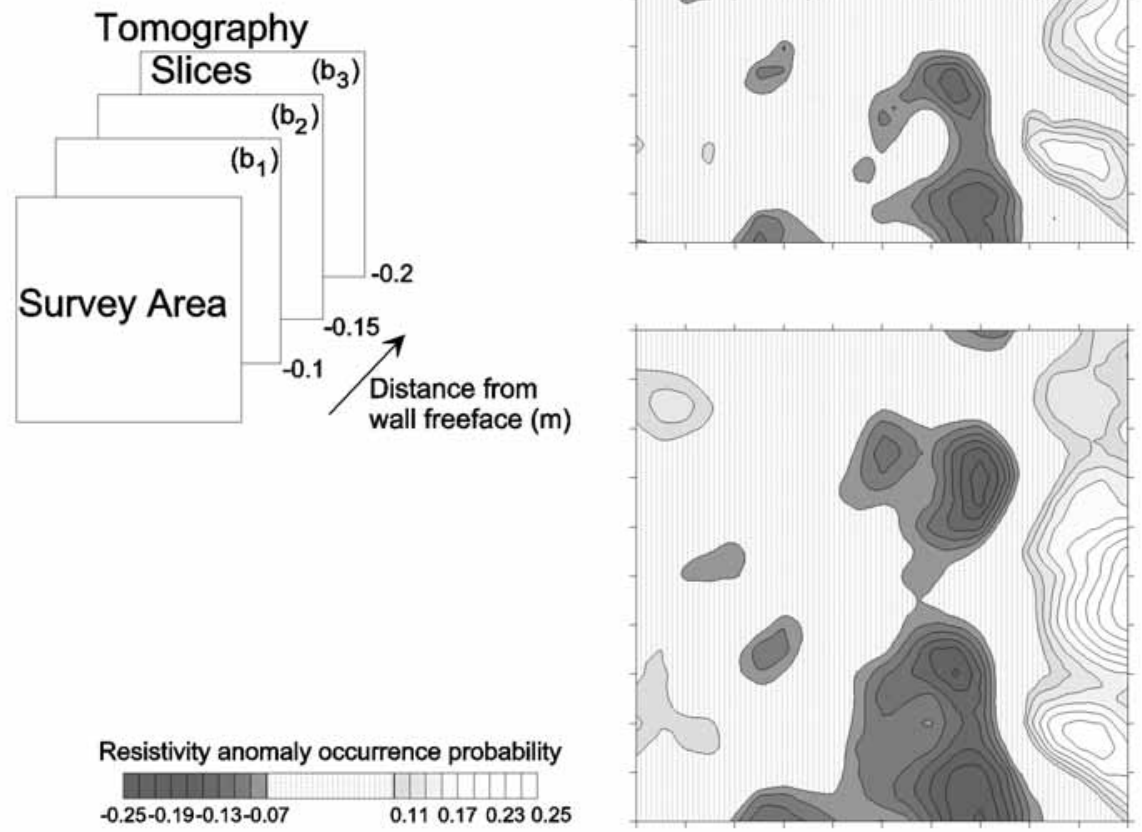

$\left(b_{3}\right.$

Fig. 7a,b. The tensor resistivity probability tomography applied to a portion of wall in the Pietra Serena Cloister at San Giovanni a Carbonara (Naples, Italy). a) The surface map of the invariant parameter related to the trace of the determinant of the apparent resistivity tensor. b) A sequence of tomographic slices at increasing penetration. 


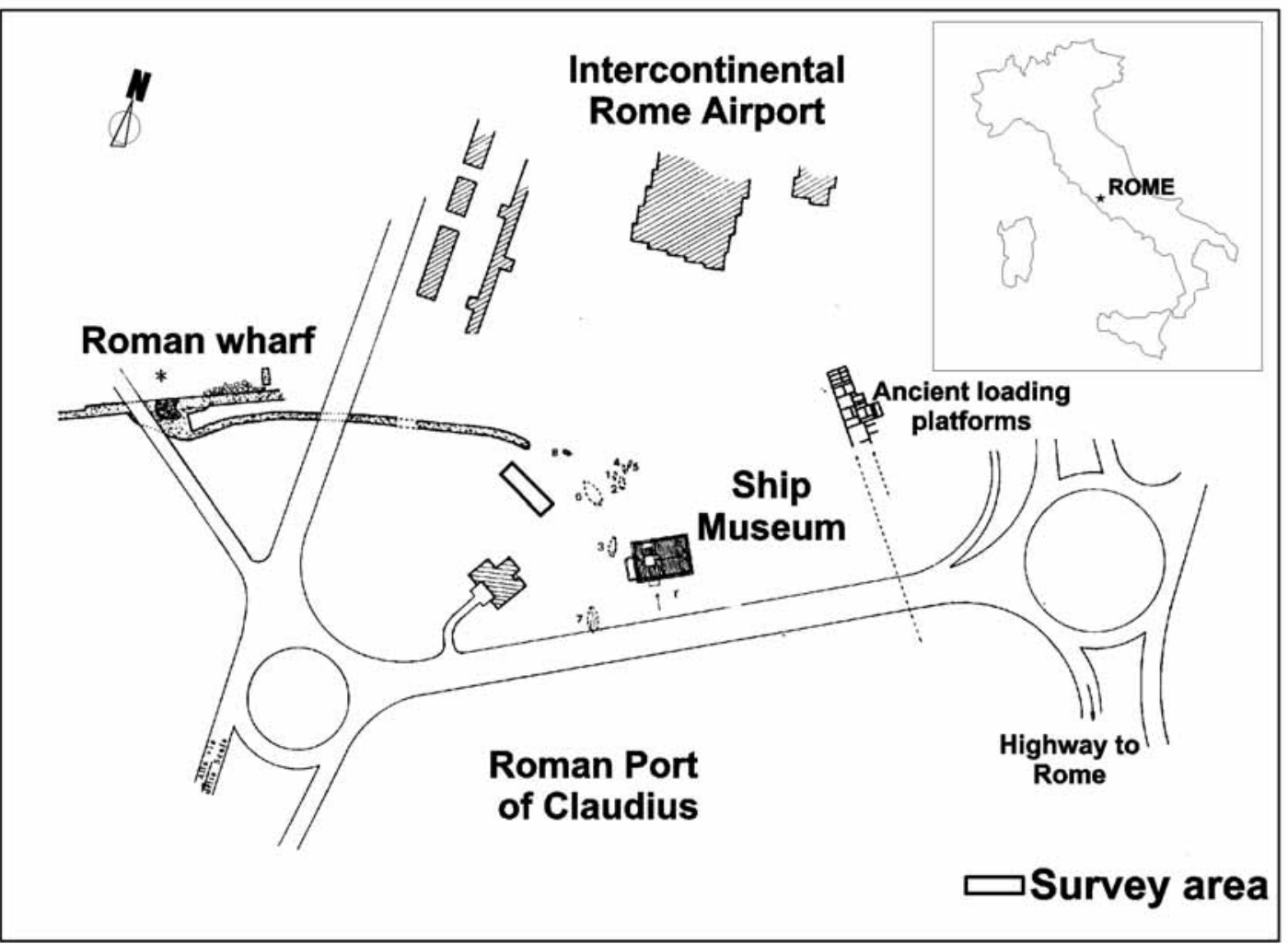

Fig. 8a,b. The archaeological site of the Roman Port of Claudius at Fiumicino (Rome, Italy). The rectangle indicates the area prospected by the geoelectrical survey.

We interpret the sequence of negative nuclei as a trace of the trench, whose refilling sediments appear less resistive than around because of major water content due to increased permeability. The positive nucleus can instead be interpreted as a possible site where marble remnants can still be dug out.

\section{Conclusions}

The examples which we have dealt with in the previous section show how effective the imaging capability of the TRPT method is in outlining buried features for architectonic and archaeological purposes. TRPT is indeed a powerful combination of two specific improve- ments made in modern high-resolution geoelectrical prospecting. The first step was the realisation of a non-invasive survey technique aimed at highlighting all the diagnostic properties of the apparent resistivity tensor, using a pair of independent current sources and measuring the two horizontal components of each generated electric field. The second step was the theoretical formulation of the probability tomography method for tensorial geoelectrics and the introduction of the RAOP function as a suitable tool for imaging the most probable locations of the physical targets, which are responsible for the observed matrix elements of the apparent resistivity tensor.

A remarkable aspect of this study is that the resistivity signatures have been disclosed only 


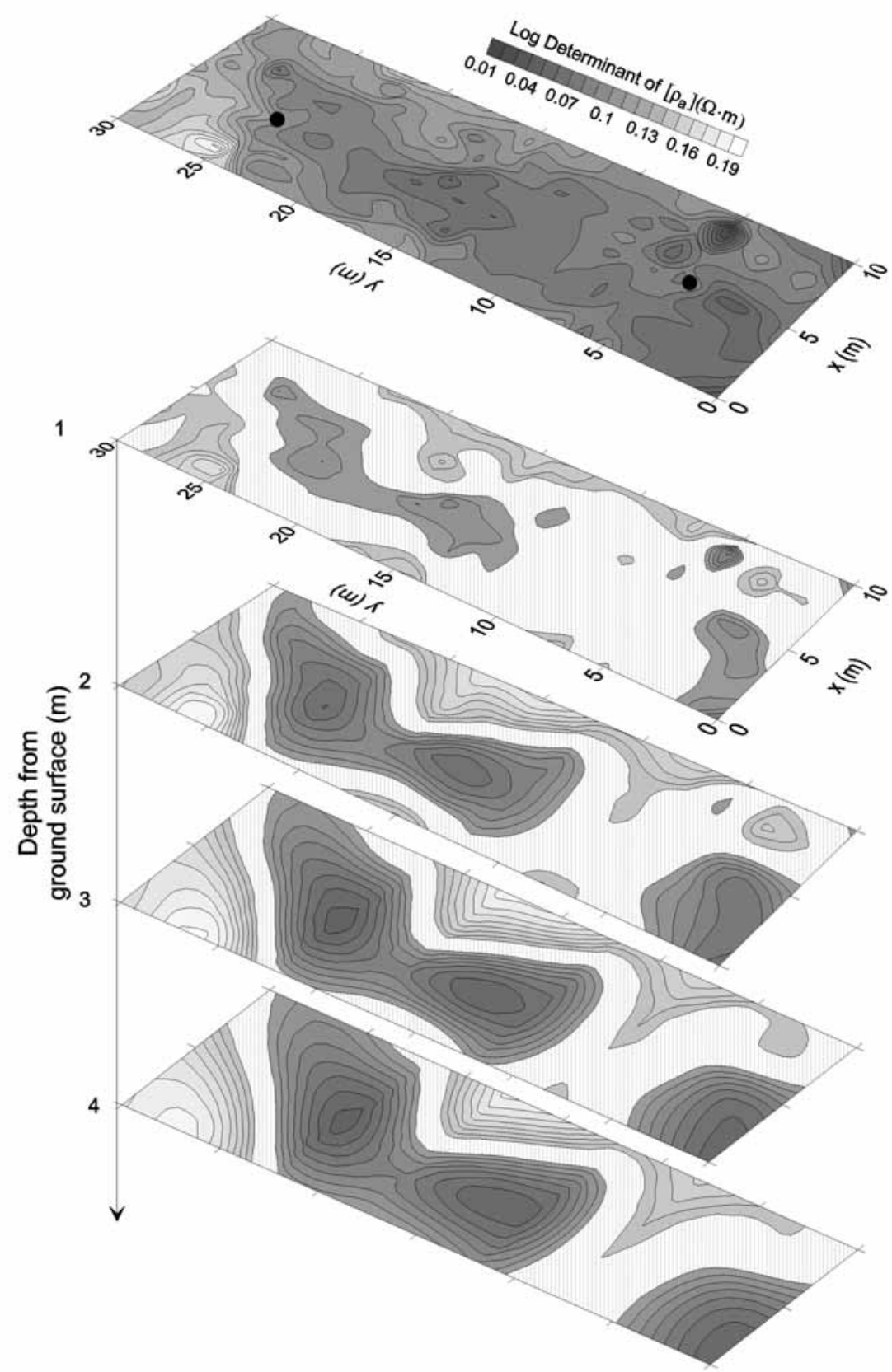

(a)

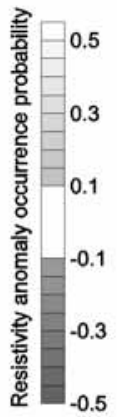

Fig. 9a,b. The tensor resistivity probability tomography applied to a portion of land in the archaeological site of the Roman Port of Claudius at Fiumicino (Rome, Italy). a) The surface map of the invariant parameter related to the trace of the determinant of the apparent resistivity tensor. The two black dots over the map indicate the places where remnants were dug out. b) A sequence of tomographic slices at increasing penetration. 
from a probabilistic viewpoint, which conforms to the inherently uncertain nature of the geophysical interpretation process. The TRPT approach is the consequence of the intrinsic nonunivocity of the geophysical solution, as many models provide, within the measuring accuracy, equivalent responses which cannot be discriminated without external constraints.

\section{REFERENCES}

BiBBy, H.M. (1977): The apparent resistivity tensor, Geophysics, 42, 1258-1261.
BIBBY, H.M. (1986): Analysis of multiple-source bipolequadripole resistivity surveys using the apparent resistivity tensor, Geophysics, 51, 972-983.

MAURIELLO, P. and D. PATELLA (1999): Resistivity anomaly imaging by probability tomography, Geophys. Prospect., 47, 411-429.

MAURIELLO, P. and D. PATELLA (2001): Introduction to multiplesource resistivity probability tomography, Geophysics (submitted).

Mauriello, P., D. Monna and D. Patella (1998): 3D geoelectric tomography and archaeological applications, Geophys. Prospect., 46, 543-570.

PATELLA, D. (1997a): Introduction to ground surface self-potential tomography, Geophys. Prospect., 45, 653-681.

PATELLA, D. (1997b): Self-potential global tomography including topographic effects, Geophys. Prospect., 45, 843-863. 